\title{
L'ergonomie didactique face au défi de la formation ouverte et à distance
}

Jean-Claude Bertin

\section{(2) OpenEdition}

\section{Journals}

Édition électronique

URL : http://journals.openedition.org/asp/1163

DOI : 10.4000/asp. 1163

ISBN : 978-2-8218-0394-7

ISSN : 2108-6354

Éditeur

Groupe d'étude et de recherche en anglais de spécialité

Édition imprimée

Date de publication : 1 novembre 2003

Pagination : 47-66

ISSN : 1246-8185

Référence électronique

Jean-Claude Bertin, «L'ergonomie didactique face au déf de la formation ouverte et à distance », ASp [En ligne], 41-42 | 2003, mis en ligne le 24 avril 2010, consulté le 19 avril 2019. URL : http:// journals.openedition.org/asp/1163; DOI : 10.4000/asp.1163

Ce document a été généré automatiquement le 19 avril 2019

Tous droits réservés 


\title{
L'ergonomie didactique face au défi de la formation ouverte et à distance
}

\author{
Jean-Claude Bertin
}

\section{Introduction}

1 Malgré des appellations différentes selon les époques et les modes (EAO, EIAO, TELL, WELL

${ }^{1}$...) les différentes orientations qui ont marqué la courte histoire de l'apprentissage médiatisé par ordinateur semblent désormais converger vers l'utilisation de l'Internet et la mise en ligne des matériaux de formation. Une première vague d'applications informatiques essentiellement locales (développements spécifiques aux institutions, systèmes-auteurs propriétaires ${ }^{2}$, logiciels éducatifs et tutoriels ${ }^{3}$ sur cédérom "prêts à l'emploi », etc.) s'est appuyée sur des expérimentations visant à mettre en œuvre, à tester ou valider une approche théorique, ou à tirer parti d'une avancée technologique (oralisation de l'ordinateur, intégration de la vidéo, de la reconnaissance de la parole, etc.), sans autre contrainte que celles impliquées par l'écriture du code informatique. Les chercheurs et utilisateurs de ces technologies ont contribué à mettre en valeur un ensemble de critères de qualité maintenant admis par la communauté. Nous avons, pour notre part, proposé un modèle d'intégration des nouvelles technologies dans l'enseignement / apprentissage des langues, fondé sur le concept d'ergonomie didactique (Bertin 2001, 2002).

2 La mise en place d'une pédagogie relayée par l'informatique requiert un investissement important en matière de finances, de temps de développement et de réflexion sur les conditions de son intégration dans la situation pédagogique. Se pose alors le problème de la «rentabilisation » des produits, c'est-à-dire de leur utilisation optimum par le plus grand nombre, et par conséquent de leur partage entre enseignants, équipes pédagogiques, voire institutions. Le succès commercial des systèmes-auteurs propriétaires s'est trouvé de facto limité par la demande de ressources facilement 
échangeables d'un lieu, d'un équipement à un autre. Rapidement, la généralisation des navigateurs Internet a offert une solution évidente aux problèmes de compatibilité des matériaux qui, une fois mis en ligne, sont devenus accessibles à tout utilisateur connecté, réglant dans le même temps les difficultés ergonomiques fonctionnelles propres à chaque environnement propriétaire.

Pour autant, si l'Internet apparaît incontournable, il ne garantit pas à lui seul la qualité pédagogique des matériaux qui y sont présentés. La mise en ligne de pages Web suppose en effet des contraintes que ne connaissaient pas les premières expérimentations en local. Si celles-ci gardaient constamment ouvert le champ de la créativité (l'informaticien programmant intégralement la machine pour répondre au cahier des charges du didacticien), l'Internet implique le passage par les fourches caudines d'un langage commun. Ce langage, dit «de description $»^{4}$, précise les normes de présentation des matériaux mis en ligne, tout en limitant l'interactivité entre l'utilisateur et la machine au seul hypertexte/hypermédia ${ }^{5}$. Malgré la richesse conceptuelle de ces hyperdocuments, le didacticien à la recherche de formes variées d'interactivité n'y trouve pas toujours son compte. Plus récemment, de véritables langages de programmation ${ }^{6}$ sont venus enrichir l'environnement $\mathrm{du}$ Web et offrir de nouvelles solutions susceptibles d'élargir les horizons descriptifs du seul html.

4 Cet enrichissement relance en même temps la distinction un temps atténuée entre « créateur » et « informaticien »: si le premier peut aisément recourir à des logiciels de création de page Web ne requérant qu'une compétence informatique limitée (Word, Dreamweaver et autres), la programmation de fonctions avancées sous-entend le plus souvent l'intervention du second...

5 La question qui se pose est de savoir comment les modèles existants peuvent trouver avec l'Internet un champ d'application nouveau, garantissant un environnement d'enseignement/apprentissage apte à satisfaire les exigences de l'enseignant de langue. La pression (institutionnelle ou financière) du e-learning et des campus numériques peut conduire à de nouvelles hiérarchies pas toujours compatibles avec ces exigences. La finalité même de ces nouveaux développements est en cause : s'agit-il simplement d'offrir par la distance un accès plus souple à l'information ou de tenter de faciliter, par des matériaux de nature plus complexe, la mise en œuvre des processus cognitifs chez l'apprenant, que les activités traditionnellement dévolues à la salle de classe suscitaient seules jusqu'alors ? La réponse à ces questions dépend étroitement des facteurs qui soustendent les processus de médiation pédagogique spécifiques à la formation ouverte et à distance (FOAD). Quels sont ces facteurs? Sont-ils compatibles avec le modèle d'intégration que nous avons proposé ? L'introduction de la distance en temps et espace invalide-t-elle ou modifie-t-elle ce modèle? Après un bref rappel des fondements théoriques auxquels nous nous référons, nous analyserons deux exemples de matériaux développés pour la formation à distance afin de mettre en évidence ces facteurs et de faire évoluer ainsi le modèle proposé. Le présent article constitue une première contribution à cette réflexion menée dans le cadre du $\mathrm{CIRTAI}^{7}$, à l'Université du Havre. 


\section{Rappel théorique : les fondements de l'ergonomie didactique}

Sans reprendre ici ce que nous avons écrit plus en détail précédemment, rappelons simplement que l'ergonomie didactique est une approche théorique de l'instrumentation technologique de la situation d'apprentissage, visant à l'adéquation entre la technologie, ses utilisateurs et le processus, c'est-à-dire l'objectif recherché (l'acquisition de nouveaux savoirs et savoir-faire). À partir des pôles de référence de la didactique, dont elle cherche à préciser l'importance, elle tente de décrire les interactions à l'œuvre dans l'enseignement/apprentissage des langues médiatisé par ordinateur, pour en déduire un contexte d'intégration optimale des outils.

7 De manière simplifiée (fig. 1), elle considère que l'ordinateur constitue le centre d'un environnement d'apprentissage virtuel, inséré dans l'environnement réel au sein duquel évolue l'apprenant, et qui regroupe enseignant, supports pédagogiques, pairs... Émerge ainsi un véritable système HommeS-Machine ${ }^{8}$ dont il convient de comprendre le fonctionnement.

8 L'un des principes essentiels de l'ergonomie didactique consiste à affirmer que le recours à la technologie se justifie lorsque le matériau pédagogique est (re)pensé en fonction de la nature spécifique du support, de ce qu'il peut apporter de nouveau et de mieux au processus visé.

Fig. 1. Représentation simplifiée du modèle d'ergonomie didactique ${ }^{9}$

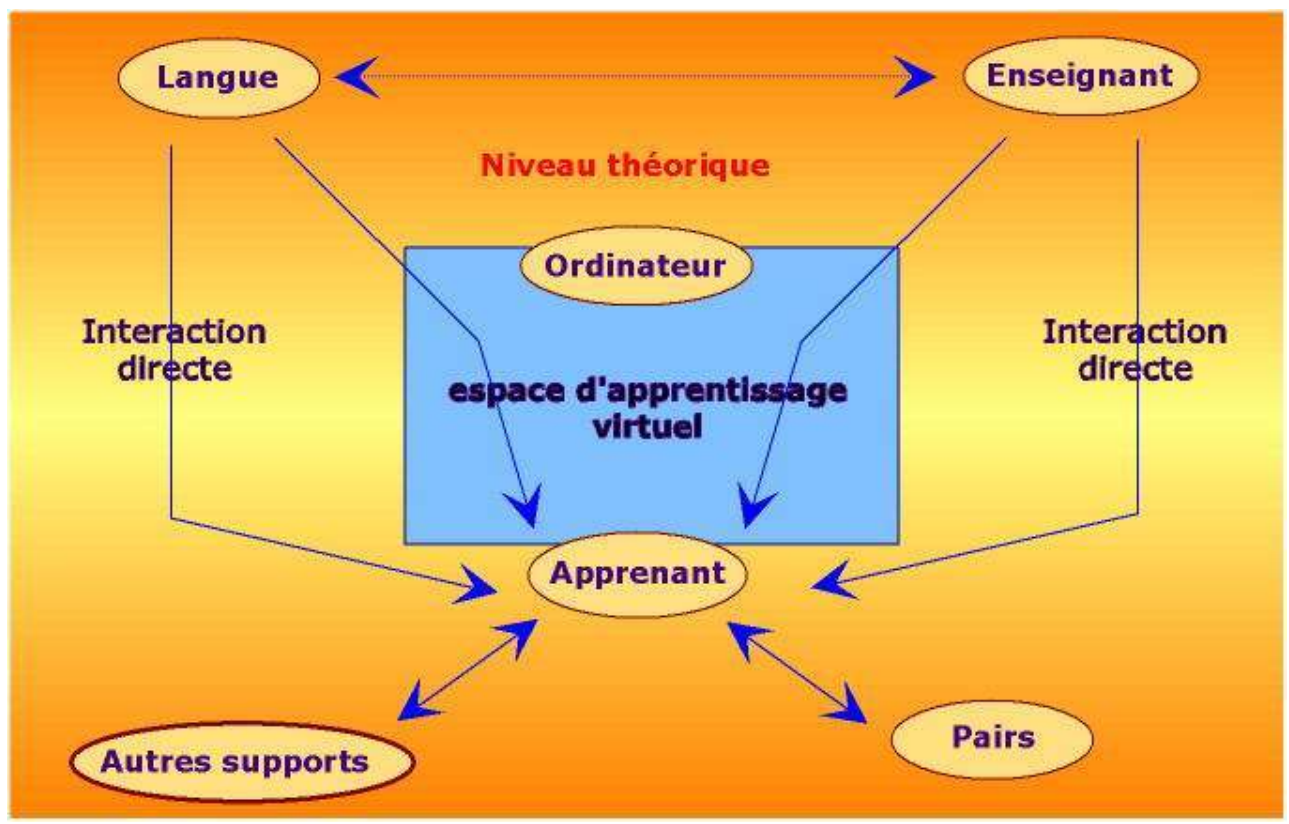

En ce sens, il serait vain de ne se tourner vers l'Internet que parce qu'il offre cette facilité d'échange et de partage des matériaux ou parce que son usage constituerait une mode censée renforcer la motivation de l'apprenant. Ce à quoi il convient de tendre est le transfert vers ce nouvel environnement de ce que les expériences antérieures ont montré être la justification profonde de l'utilisation pédagogique de l'ordinateur. 

l'interdépendance est facteur de qualité, et dont la combinaison constitue ce que nous avons appelé " ergonomie didactique ». Les fig. 2, 3 et 4 rappellent succinctement la nature de ces sous-systèmes, qui seront utilisés comme points de départ pour l'étude présentée dans les pages qui suivent.

11 La relation enseignant-langue-ordinateur (fig. 2. Ergonomie pédagogique) détermine les conditions de création des matériaux pédagogiques, qui dépendent à la fois de la nature de la langue-objet, des contraintes technologiques (capacité de la machine à reproduire la langue) et de la créativité offerte par les caractéristiques du support (richesse de l'interactivité, par exemple).

Fig. 2. Sous-système enseignant-langue-ordinateur = ergonomie pédagogique

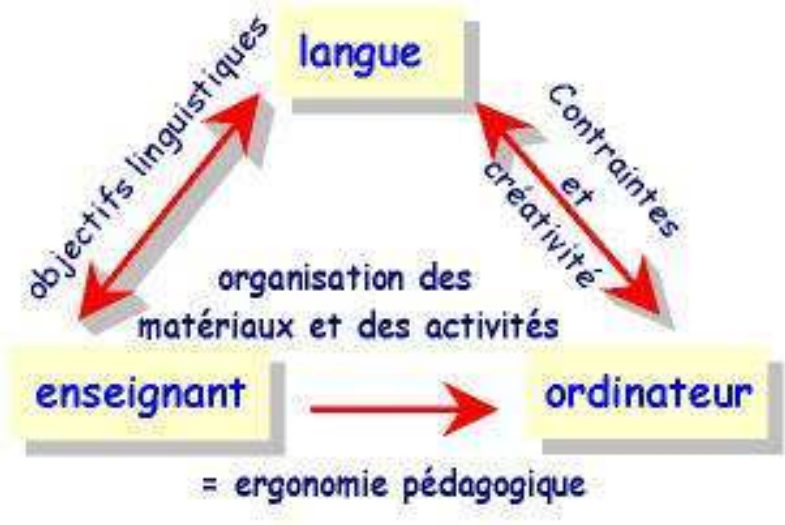

12

La relation apprenant-langue-ordinateur (fig. 3. Ergonomie cognitive) correspond à l'environnement d'apprentissage virtuel, « préparé » en amont (en partie tout au moins) par l'enseignant, mais actualisé par l'apprenant dans son interaction avec l'ordinateur. Ergonomies pédagogique et cognitive se situent dans un rapport temporel.

Fig. 3. Sous-système apprenant-langue-ordinateur = ergonomie cognitive

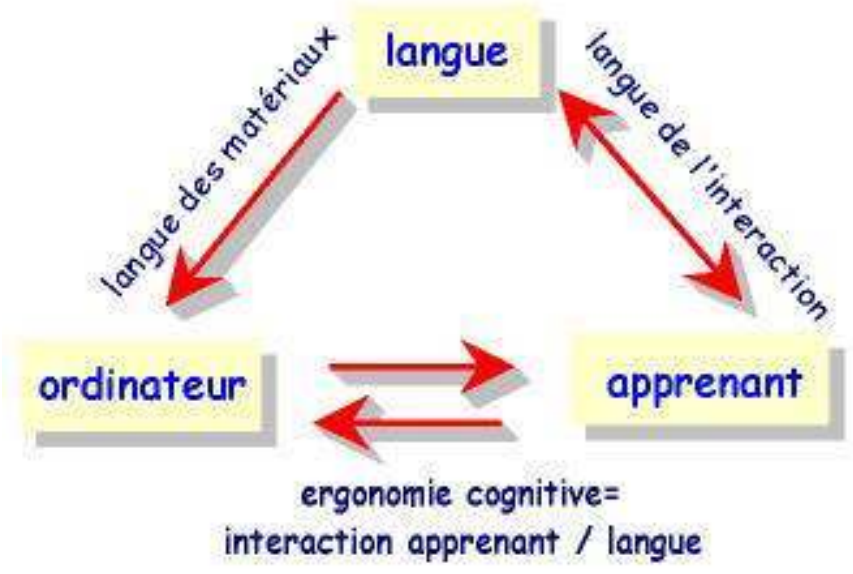

La relation enseignant-ordinateur-apprenant (fig. 4. Suivi pédagogique) est fondée sur la capacité de la machine à enregistrer les actions de l'apprenant en réponse aux stimuli, 
puis à transmettre ces informations à l'enseignant, qui pourra, en toute connaissance de cause, préparer de nouveaux matériaux pour des activités de remédiation, de renforcement...

Fig. 4. Sous-système enseignant-ordinateur-apprenant = suivi pédagogique

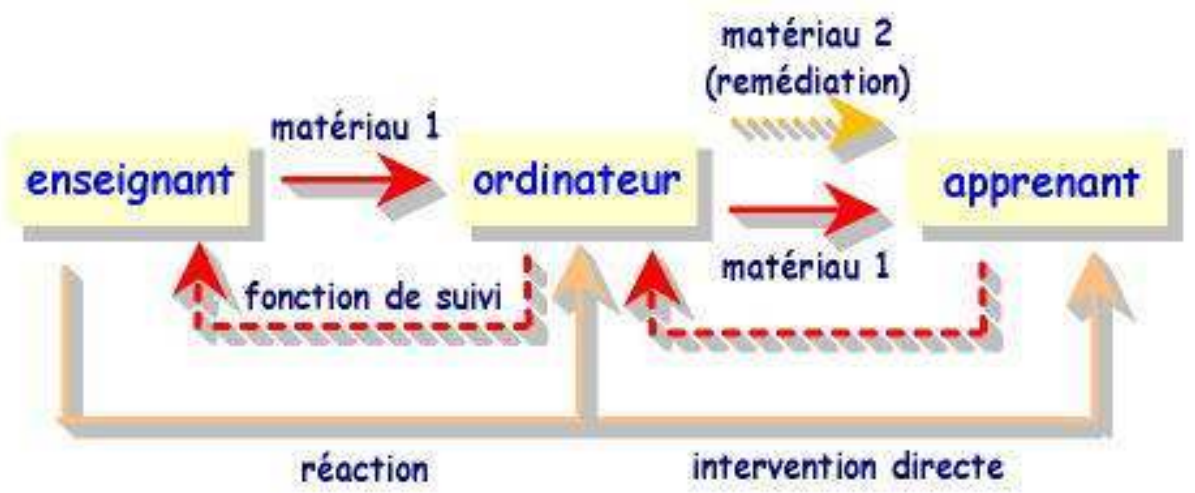
de matériaux en ligne, développés dans des contextes distincts. À travers cette diversité (de publics, d'objectifs et de disciplines), nous tenterons d'observer quelles convergences apparaissent et comment le modèle ergonomique, dont pourra s'inspirer en particulier le linguiste, s'en trouve validé ou modifié.

17 Notre premier exemple sera le campus numérique Pegasus ${ }^{10}$, développé dans le cadre d'un consortium regroupant les Universités d'Antilles-Guyane, Haute Alsace, Le Havre, Paris 13, Nice Sophia-Antipolis, Toulon-le Var, ainsi que le CNED. L'objectif en est la préparation au DAEU ${ }^{11}$, et son public relève majoritairement de la formation continue. Ce campus est opérationnel depuis cette année. L'objectif de ces matériaux, maintenant opérationnels, est la préparation au DAEU : français, mathématiques, anglais, histoire, géographie.

18 Le second exemple est plus expérimental : il est le fruit du programme européen LET'S ${ }^{12}$ ( Learning European Transport Sciences), mené sur fonds Socrates, dont l'objectif était de rendre accessibles un certain nombre de matériaux pédagogiques développés dans le 
cadre du European Master of Transport ${ }^{13}$. Ce diplôme se prépare sur deux semestres effectués dans deux des cinq universités européennes participant au programme. Il s'agit des universités du Havre, pour deux modules (Langues du Transport, Aménagement), de Santander (Mobilité et Logistique Intégrale), de Liège (Analyse des Systèmes de Transport), de Gand (Droit du Transport) et de Dresde (Management Logistique). À partir d'un cahier des charges commun, reflétant les objectifs généraux du Master, chaque université devait développer des matériaux apportant un complément pédagogique aux modules qu'elle dispense, tout en conservant une liberté de manœuvre appréciable afin de faire face aux situations locales ainsi qu'au degré d'expertise variable dans le domaine de l'ingénierie pédagogique multimédia.

Les deux exemples retenus ont en commun le fait d'être pluridisciplinaires, mais se distinguent principalement par le fait que le premier répond à un cahier des charges plus contraignant que le second et que la préparation au diplôme du premier s'effectue totalement à distance, alors que le second vise uniquement à mettre en ligne un complément de formation.

L'analyse de ces deux séries de matériaux pédagogiques fait apparaitre des divergences significatives dans les processus de médiation des connaissances pour une formation à distance. À première vue, certaines habitudes culturelles (place accordée au texte écrit dans la communauté des juristes, par exemple) semblent prédominer dans la conception des matériaux et s'opposer parfois à des critères d'ergonomie fonctionnelle, voire didactique.

La culture disciplinaire peut également être envisagée sous la forme du modèle transmissif habituellement retenu: les juristes s'inscriraient alors dans une perspective d'enseignement (enseignant 痗 discipline 嵁 apprenant) alors que linguistes et géographes se rejoignent dans une approche alliant constructivisme et cognitivisme, centrée sur l'apprentissage (apprenant 爁 discipline 殹 enseignant) ${ }^{14}$.

On observe ainsi une dichotomie entre deux grands types de disciplines, l'un visant à transmettre des savoirs (le droit du transport, par exemple), l'autre plus préoccupé par le développement de savoir-faire chez l'apprenant (c'est le cas par exemple des langues dans le projet LET'S).

Ces approches débouchent sur des matériaux divergents dans la nature des approches didactiques suivies, le choix des contenus et des modes d'utilisation, la sélection ou non de plateformes spécifiques, l'ergonomie générale du système.

\section{Finalités des matériaux en ligne}

De manière synthétique, on pourrait décrire les matériaux étudiés comme la combinaison des facteurs représentés dans la fig. 5 . Chaque élément de ce système complexe entretient d'étroites relations avec les autres, ouvrant ainsi un champ d'interactions que doit tenter de maîtriser le concepteur. Selon le cas, ces interactions apparaissent comme autant de contraintes ou, à l'inverse, de sources de créativité.

La conception de matériaux en ligne suppose une démarche par étapes successives de conception, de tests, suivies de boucles de rétroaction qui introduisent dans la maquette initiale le résultat des observations en situation. 
Dans la fig. 5, les flèches grises indiquent les facteurs qui interviennent dans la phase initiale de conception. Les flèches noires marquent les relations qui s'instaurent entre ces facteurs lors des phases d'observation et de test et qui induisent des aménagements au sein des matériaux.

Fig. 5. Facteurs influençant directement la création de matériaux pédagogiques en ligne.

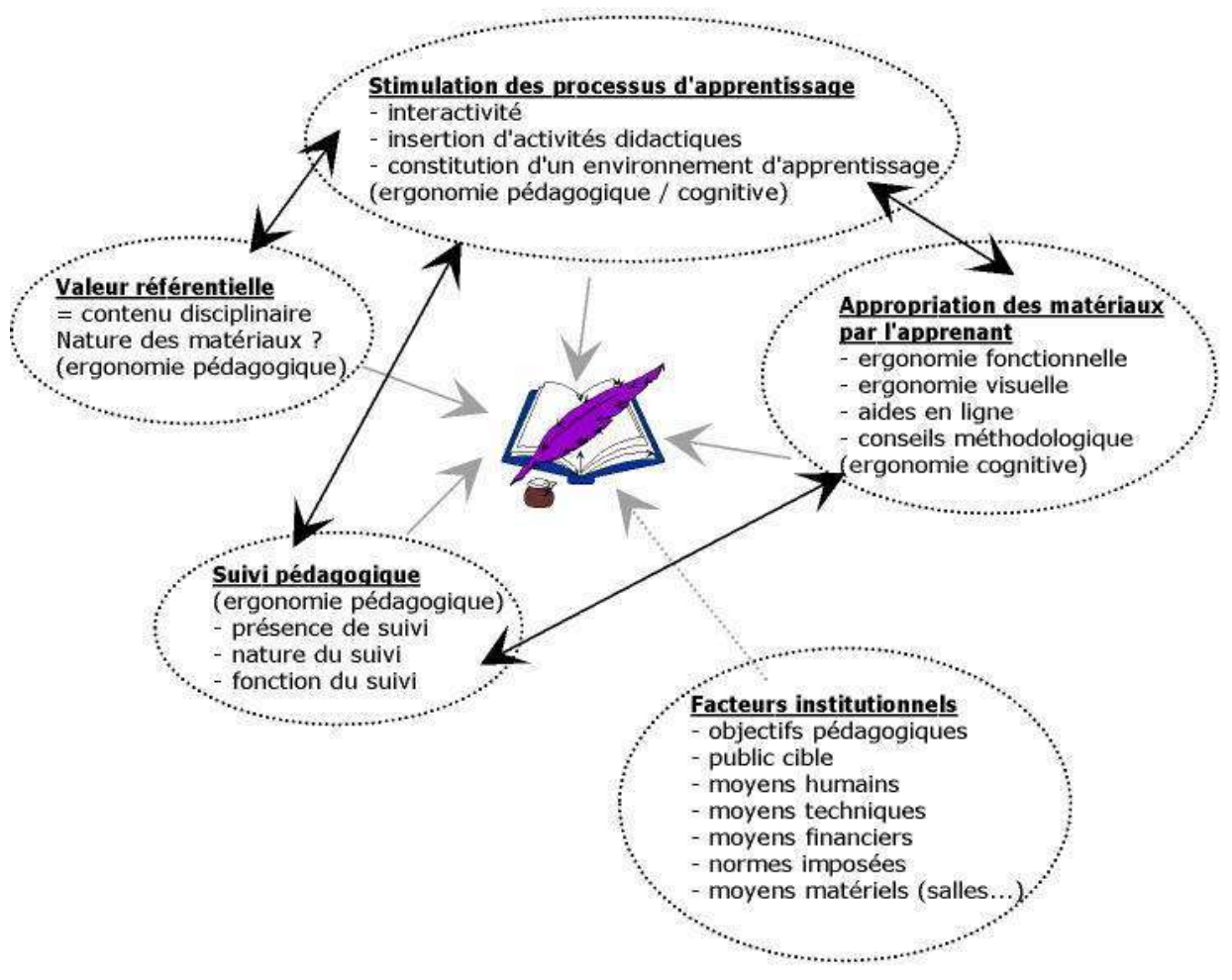

\subsection{Finalité référentielle des matériaux}

Cette première finalité relève du champ de l'ergonomie pédagogique et est directement liée au domaine disciplinaire. Le matériau en ligne est censé apporter un contenu à l'apprenant ${ }^{15}$, contenu dont la nature varie d'une discipline à l'autre.

De ce point de vue, la perception des relations existant entre l'ordinateur et ses utilisateurs varie de manière significative dans les exemples étudiés. Cette variation est particulièrement visible dans le programme LET'S, pour lequel aucune norme formelle n'était imposée au départ.

On pourrait ainsi ranger les matériaux étudiés sur un axe dont une extrémité serait constituée par ceux dont la finalité principale est de rendre accessibles à distance des documents de référence, porteurs des savoirs à acquérir. Le support numérique remplace ici le polycopié utilisé dans une pédagogie traditionnelle et présentielle, et rejoint l'approche "classique» d'une formation à distance fondée sur l'envoi de cours sur support papier.

On ne s'étonnera pas de trouver cet objectif au centre de la démarche de Pegasus, à la conception duquel le CNED a activement participé, transférant ainsi son savoir-faire vers le support informatique. Tous les documents de travail autour desquels s'articulent les scénarii pédagogiques (toutes disciplines confondues, langues comprises) sont 
téléchargeables sous la forme de fichiers .pdf reprenant le format des polycopiés traditionnels.

Plus caractéristique encore est le cas du module de droit du transport dans le programme LET'S. Le « cours » en ligne correspond à la simple numérisation des textes de référence : il semblerait que le terme « cours » en lui-même ait été compris par les juristes dans son acception minimale (c'est-à-dire le contenu du cours, habituellement publié par cette université sur support papier pour les étudiants) et non dans celle, plus large, adoptée par d'autres partenaires (c'est-à-dire le processus pédagogique complet par lequel les savoirs ou savoir-faire sont transmis par l'enseignant et assimilés par l'apprenant).

La finalité référentielle du matériau peut toutefois être plus floue, lorsque l'objectif visé est moins la transmission de savoirs que le développement de savoir-faire spécifiques.

Ainsi, le module Aménagement de LET'S tire profit des liens hypermédias pour proposer un contenu disciplinaire d'un abord moins dense, sur le plan de l'ergonomie visuelle, que l'apprenant (re)constitue au fur et à mesure de son hypernavigation au sein du document.

Fig. 6. Module Aménagement (LET'S) - finalité référentielle et hypermédia

\section{I- LA POLITIQUE DES TRANSPORTS DE L'UE}

I-1 Une politique anormalement tardive (1987-1986)

réf : fiche documentaire $\mathrm{n}^{\circ} 1$, Les grandes étapes de la politique des transports

I-2 Les résultats acquis ( 1986-2000)

L'harmonisation, le cabotage ( ref : fiche documentaire $n^{\circ} 1$ )

I-3 Un nouveau chantier : les Réseaux Transeuropéens de Transport

réf : fiche documentaire $\mathrm{n}^{\circ} 2$, La carte des projets RTT

Exercices sur la première partie

Retour haut de page

Par ailleurs, la finalité référentielle semble directement influer sur la nature des documents proposés : texte simple, adjonction d'images (illustrations, cartes et autres graphiques), de séquences sonores ou vidéo, insertion de liens dynamiques hypertextes ou hypermédias.

Les normes adoptées pour le campus Pegasus limitent les matériaux aux seuls texte et éléments graphiques. Des raisons techniques et pédagogiques, sur lesquelles nous reviendrons, expliquent ces choix.

Les matériaux développés dans le programme LET'S utilisent quant à eux toute la gamme des médias disponibles, mais de manière sensiblement différente selon les disciplines :

- droit, management logistique, analyse des systèmes de transport : texte seul ;

- aménagement, logistique intégrale : texte, majoritairement, et image;

- anglais du transport : texte, image, son, vidéo. 


\section{la nature des documents utilisés, on peut penser qu'il s'agit de l'influence de la «culture} disciplinaire » (habitudes pédagogiques et références admises dans le champ).

Cette culture est particulièrement visible, par exemple, dans le choix de documents juridiques essentiellement monomédias, dans leur version originale. Bien que quelques liens dynamiques (pour les notes bibliographiques, notamment) aient été rajoutés ultérieurement, ils restent hypertextuels (absence de multi- ou hyper-média). Ceci correspond typiquement tant à une culture pédagogique accordant une large place à l'usage du polycopié qu'à une culture juridique où le mot écrit prime sur toute autre forme de communication ${ }^{16}$.

39 A l'inverse, la préparation à la communication orale impliquée par la spécialisation en langue du transport sous-entend la rédaction de documents multi- et hypermédias. L'absence remarquable de tels documents dans la préparation en langue de Pegasus s'explique en partie par l'objectif de la formation actuellement dispensée (le DAEU), qui ne requiert qu'une évaluation écrite de la langue.

\subsection{Stimulation des processus d'apprentissage}

40 À l'autre extrémité de l'axe que nous avons défini se situent des matériaux visant essentiellement à stimuler les processus d'apprentissage. Plus fondés sur le développement de savoir-faire que sur la seule transmission de savoirs, ceux-ci impliquent parfois de repenser totalement la pédagogie, pour tirer un profit spécifique de la technologie multimédia et hypermédia (le module d'anglais du transport de LET'S est une tentative en ce sens).

41 Entre ces deux extrêmes, les deux sites étudiés proposent une gamme de matériaux reflétant les différentes formes que peut revêtir le concept d'interactivité :

- nombre plus ou moins élevé de liens hypertextes (références bibliographiques, par exemple, pour le management logistique);

- enrichissement des documents par des liens hypermédias (cartes pour le module aménagement) ;

- insertion d'activités didactiques (simulations, pour le module analyse des systèmes de transport, ou exercices - norme requise pour tous les modules Pegasus).

42 Le caractère interactif des matériaux étudiés semble répondre principalement à l'une ou l'autre de deux fonctions distinctes.

\subsubsection{L'interactivité pour la construction du savoir}

Les matériaux sont organisés autour d'un ou plusieurs documents majeurs, complétés d'un nombre variable de documents accessibles par l'intermédiaire d'un menu ou reliés par hypertexte.

Citons le module aménagement de LET'S (fig. 6), centré sur un hyperdocument à partir duquel l'apprenant accède, par un jeu de liens hypermédias, à des fiches documentaires ou méthodologiques, qu'il active ou non selon ses besoins individuels et ses stratégies.

Un autre exemple caractéristique nous est proposé par la structure des modules Pegasus, centrés autour d'un "polycopié» en format pdf, complété par un jeu de documents accessibles à partir d'un menu situé en tête de page (fig. 7). 
Fig. 7. Campus Pegasus et construction du savoir

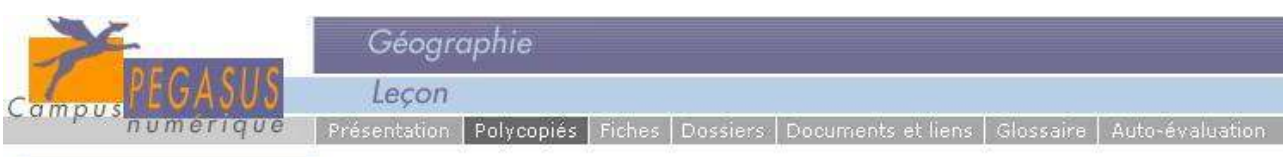
mais surtout affaire d'organisation de ces informations. Le savoir virtuel, disséminé de manière informe dans le matériau hypermédia, doit devenir formel et organisé selon des méthodes que l'apprenant doit apprendre à reconnaitre: il s'agit là de l'apport des théories cognitivistes. Pegasus ne s'y est pas trompé en proposant un module complet - et obligatoire - sur la manière d'apprendre à distance. en plein essor sur l'Internet, des Webquests, qui proposent sur un même site un ensemble de liens dédiés destinés à aider l'apprenant dans le processus de résolution des problèmes qui lui sont soumis.

53 Citons enfin les nouvelles maquettes logicielles pour l'anglais du transport, construites selon la perspective actionnelle mise en exergue dans le Cadre Européen Commun de Référence (2001). Il s'agit ici de dépasser les compétences uniquement langagières et 
d'engager l'apprenant dans une tâche globale pour former non seulement des interlocuteurs, mais des acteurs sociaux. Les matériaux récents développés au Havre cherchent à mettre l'apprenant en situation, grâce à un bureau (fig. 8) à partir duquel il peut accéder à un ensemble de documents (textuels, sonores, vidéos, sites Internet) lui permettant de rechercher les informations utiles à la tache confiée à l'ensemble du groupe.

Fig. 8. Le bureau virtuel de l'apprenant (anglais du transport)

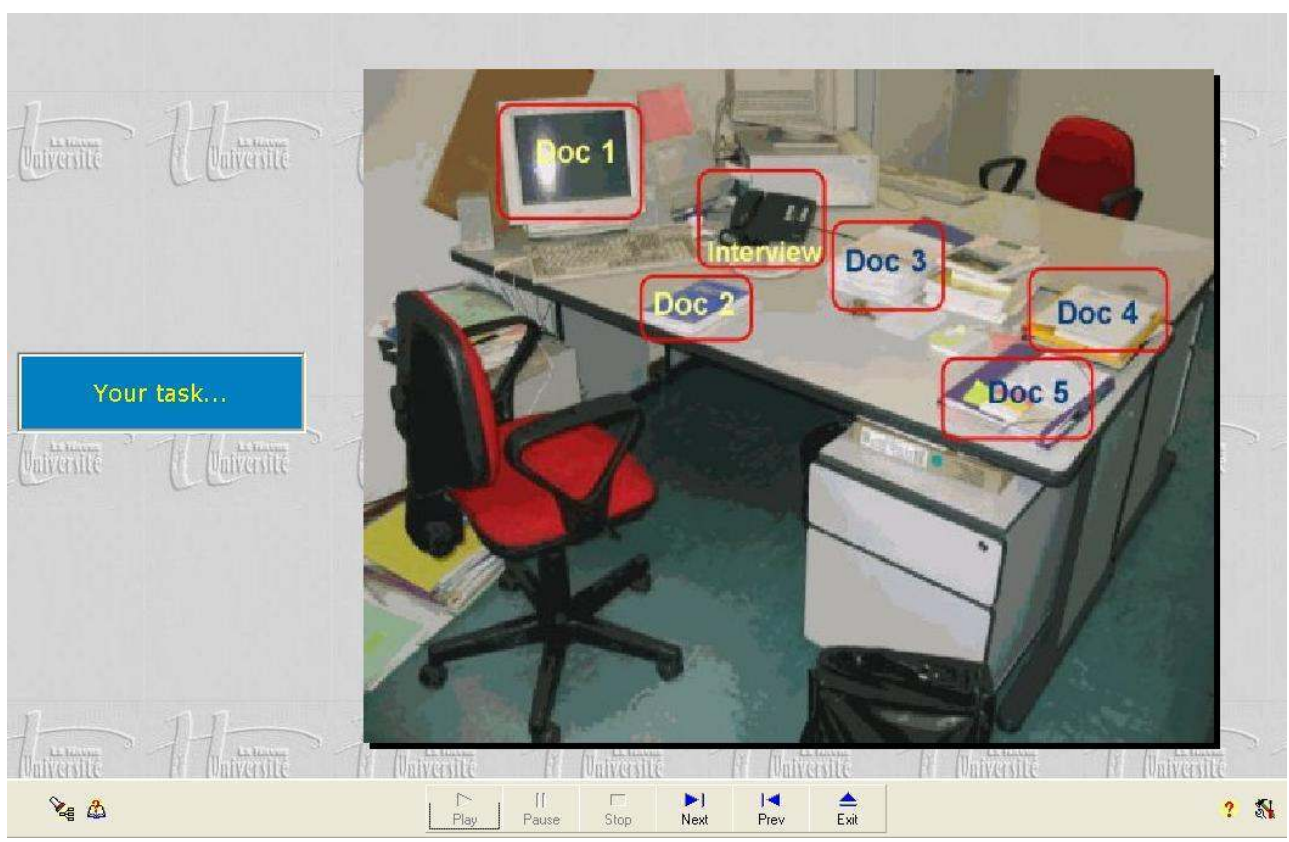

\subsubsection{L'interactivité pour la vérification/l'évaluation du processus d'acquisition}

Des phases interactives sont insérées dans les matériaux afin de s'assurer de la bonne compréhension d'un point jugé nécessaire ou de l'acquisition d'un savoir particulier, d'une technique.

La volonté pédagogique de l'enseignant concepteur est plus ou moins marquée selon les cas. On note ici encore une forte corrélation entre les matériaux de nature monomédia et à finalité référentielle élevée (le droit, par exemple) et l'absence d'activités interactives à finalité évaluative (qu'elle soit formative ou sommative). La majorité des matériaux étudiés utilise cependant cette technique pédagogique, dans des proportions et selon des modalités variables, toutefois. Nous y reviendrons plus bas lorsque nous traiterons des fonctions d'évaluation de l'apprentissage.

Nous distinguons deux types majeurs d'activités :

- l'activité est entièrement gérée par l'ordinateur: elle s'effectue intégralement à l'écran selon un processus d'interaction directe fondé sur le modèle question-réponse (ex.: qcm, exercices lacunaires) ou sur la simulation (calculs...);

- l'activité s'effectue " off-line " : la tâche et les directives afférentes sont proposées à l'écran. L'apprenant doit la réaliser selon des modalités plus traditionnelles (ex.: dessin ou annotation d'une carte), avec ou non le recours à la richesse documentaire du Web. 


\subsubsection{Structure pédagogique des matériaux}

- des documents individuels de référence ou de travail sont mis à disposition de l'apprenant, qui les consulte selon un cheminement, des objectifs et des motivations qui lui sont propres. Ces matériaux constituent généralement des appoints au cours en présentiel (droit du transport ou management logistique, dans le programme LET'S).

- les matériaux sont organisés en séquences pédagogiques selon des scénarii définis en fonction de la démarche didactique qui les sous-tend (Pegasus / module LET'S d'anglais du transport).

Cette structure pédagogique, dont deux exemples apparaissent en fig. 9 et 10, vise à guider l'apprenant de manière implicite ${ }^{18}$, en renforçant la présence de l'enseignant virtuel.

Fig. 9. Structure pédagogique du campus Pegasus

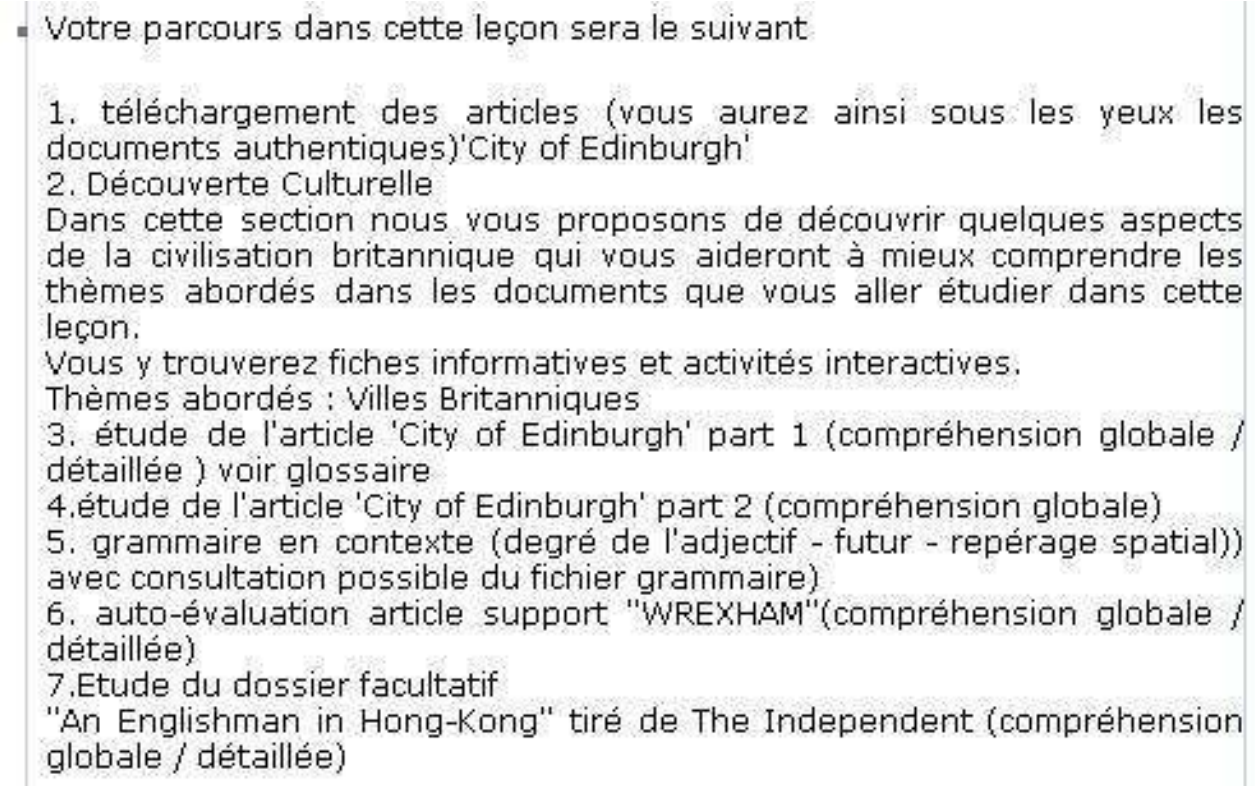


Title page

Introduction

Phase 1 : inference

Phase 2 : discovering the document

Presentation of the task / note-taking

Phase 3 : work from your notes

- MCQ : understanding meaning

Gap : memorizing vocabulary

Phase 4 : access to written text

Read and complete your Dictionary

Phase 5: oral work

Phase 6 : task - writing time

- Closing page

Il semble dans nos exemples que cette dernière option soit plutôt le fait de concepteurs bénéficiant d'une expérience de la pédagogie médiatisée par ordinateur plus grande que les autres.

\subsection{Appropriation des matériaux par l'apprenant}

Pour être efficace, le matériau en ligne doit être directement assimilable par l'apprenant, tant dans son mode de fonctionnement et d'utilisation (ergonomie fonctionnelle) que dans la représentation des connaissances qui lui est proposée (ergonomies visuelle et cognitive).

61 L'originalité majeure de la formation à distance tient précisément à cette distance imposée entre apprenant et enseignant, qui enlève au second la possibilité de réagir immédiatement aux sollicitations du premier. Les directives doivent être claires, explicites, voire redondantes, car l'apprenant se découragera vite devant un mode opératoire ou un exercice difficilement compréhensible. C'est là une condition essentielle pour éviter l'écueil du taux élevé d'abandon relevé dans les formations à distance classiques.

Fortement imprégné de l'expérience du CNED, le campus Pegasus porte une attention particulière à ce point, en proposant un module spécifique sur la méthodologie d'apprentissage à distance, mais également en prévoyant des aides interactives pour chaque activité. Un assistant animé (fig. 11.) apparaitt ainsi automatiquement à l'écran dès que l'apprenant entame une nouvelle activité afin de lui proposer un mode d'emploi en ligne. 
Fig. 11. Aide - l'agent pédagogique de Pegasus

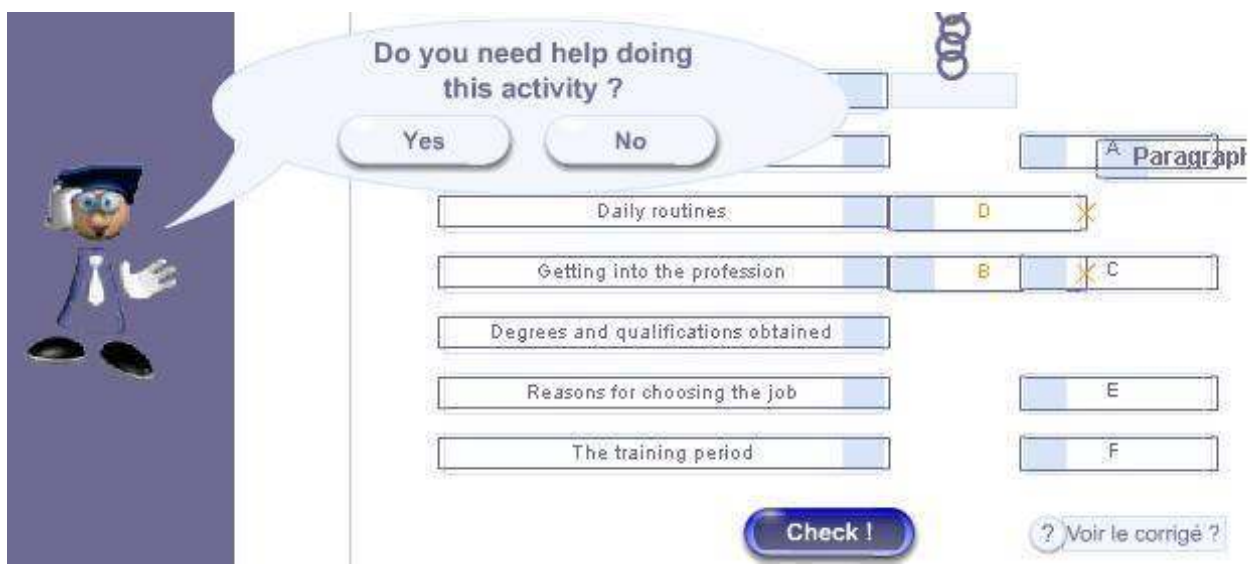

Il s'agit ici d'une variation sur le concept d'agent pédagogique (teaching agent) cher à Phillip Hubbard (Hubbard 1999, 2000). Par son graphisme, sa conception et son mode d'apparition dynamique et à-propos, ce tuteur virtuel, dont on retrouve l'icône pour toutes les fonctions d'aide et de conseil, semble bien revêtir certaines caractéristiques que Hubbard juge essentielles :

- une présence physique et une personnalité ;

- une expertise dans le domaine de référence ;

- une aptitude à l'enseignement individualisé ;

- une capacité à initier l'apprentissage.

Par son apparence et son mode opératoire, il s'agit d'une forme de médiation technologique de la présence humaine, que l'on pourrait aisément décliner selon des usages divers :

- aide à l'utilisation des activités et du logiciel ;

- conseils méthodologiques pour apprendre mieux / apprendre à distance ;

- correction des exercices proposés ;

- fourniture d'aides ponctuelles (dictionnaires ou encyclopédies...) ;

- aides à la production et à la rédaction (thesaurus pour les langues, par exemple) ;

- fourniture de références complémentaires (« pour en savoir plus »).

À la fois pédagogue ${ }^{19}$, expert et ami, l'agent pédagogique est disponible à tout moment tout en restant libre de toute connotation évaluative, à la différence de l'enseignant.

Nous remarquons toutefois une contradiction qui reste encore à lever, entre la simplicité d'utilisation des matériaux, requise par le principe d'ergonomie fonctionnelle, et ce que nous nommons «la profondeur pédagogique » des activités, c'est-à-dire la nature et la richesse des interactions mises en œuvre suite à une réponse de l'apprenant.

Pour des raisons de facilité et de rapidité de création et d'utilisation, sans parler de coût, le recours à des systèmes-auteurs de type Hot Potatoes s'est rapidement développé, malgré une profondeur pédagogique relativement réduite. Le recours à des modules d'activités plus élaborés sur le plan de leur conception didactique ne rend cependant pas toujours leur utilisation aussi aisée qu'on le souhaiterait pour l'apprenant. L'expérience en situation avec les modules Learning Labs (anglais du transport dans LET'S) révèle l'utilité d'une phase d'explication préalable des fonctions de ce produit. Le recours à un agent 
pédagogique, non prévu dans la maquette initiale, s'est avéré nécessaire pour chaque activité.

\subsection{Suivi pédagogique} concepteurs du site "Pick and Write Away $\|^{21}$ : pour dépasser les limites de la correction par l'ordinateur de la production écrite, Brett Johnson propose une correction humaine, par l'enseignant, sous forme d'annotations hypertextuelles des travaux. Ceux-ci sont ensuite remis en ligne pour le bénéfice de tous, plutôt que renvoyés par courriel, de manière confidentielle. Interrogé sur la somme de travail impliquée par un nombre important de soumissions, Brett Johnson nous a toutefois précisé qu'il était devenu nécessaire de créer une base de commentaires et d'annotations standardisés, dans laquelle peuvent puiser les correcteurs. 


$$
\begin{aligned}
& \text { encore limitée, met en évidence l'importance du tutorat pour les formations à distance, } \\
& \text { ce que tendent également à confirmer les premières observations effectuées sur } \\
& \text { l'utilisation du module d'anglais du transport. }
\end{aligned}
$$
Ce tutorat peut revêtir différentes formes :

- tutorat à distance pour un suivi, des conseils, des encouragements ;

- tutorat en présentiel, comme le pratique par exemple Pegasus, par le biais de conventions signées avec des instances locales (le réseau d'Ateliers Pédagogiques Personnalisés dans ce cas), fournissant à la fois accès aux ordinateurs et présence de tuteurs aptes à répondre en temps réel aux sollicitations des apprenants.

L'équipe de Pegasus voit un lien direct entre la mise en place de ce tutorat et un taux d'abandon beaucoup plus faible que dans les formations à distances traditionnelles. Il devient dès lors nécessaire, dès la phase de conception des matériaux, de prendre en compte la réalité et la nature du couple Homme-Machine autour duquel s'articule la formation à distance.

\subsection{Facteurs institutionnels}

81 En marge des facteurs relevant directement de l'ergonomie didactique, les facteurs institutionnels constituent le cadre dans lequel s'inscrit le processus de médiatisation de l'enseignement/apprentissage à distance.

ASp, 41-42 | 2003 
82 Annoot et Bertin (1997) ont déjà souligné le rôle joué par l'institution dans le processus d'intégration des nouvelles technologies au sein des formations en langue. L'étude des matériaux auxquels nous nous référons ici précise l'impact de plusieurs de ces facteurs sur la mise en œuvre de formations à distance.

\subsubsection{Objectif pédagogique et public cible}

83 Il s'agit de données servant de point de départ à la démarche de mise en ligne. Ces données constituent l'ossature du cahier de charge auquel doivent répondre les matériaux.

84 Dans notre étude, Pegasus est clairement identifié par rapport à ces données puisque l'objectif annoncé est la préparation du DAEU pour un public annoncé sur le site. Rappelons que ceci est l'une des raisons invoquées pour l'absence de toute oralisation des matériaux de langue, la passation de l'examen ne se référant qu'à la langue écrite. De surcroît, l'ensemble du dispositif est orienté vers l'évaluation finale (en présentiel, notons-le au passage) et constitue principalement un entraînement aux épreuves.

Le programme LET'S ne vise pas à une qualification diplômante, qui existe par ailleurs en mode présentiel, mais se présente comme un ensemble de matériaux considérés comme un complément aux formations dispensées. Ceci explique en partie le choix délibéré de certains auteurs de n'utiliser que des supports à l'interactivité réduite.

\subsubsection{Moyens humains et financiers}

Les moyens humains et financiers que permet ou non l'engagement de l'institution dans le projet déterminent à leur tour les solutions techniques. Selon le cas, le travail de conception et de développement s'effectue en équipe, avec le concours d'informaticiens chevronnés. On aura alors recours à une véritable programmation informatique ouvrant de larges horizons à la créativité des auteurs. Tel est, par exemple, le cas du module d'analyse des systèmes de transport, pour lequel il a fallu concevoir, sur le plan du code et de l'architecture des systèmes, un logiciel de simulation utilisant les ressources informatiques de l'Université de Liège.

Dans d'autres cas, l'absence d'informaticiens est palliée par le recours à un système auteur permettant à l'enseignant concepteur de réaliser seul les matériaux dont il a la charge (cas de l'anglais du transport, avec le module expérimental de mise en ligne des matériaux créés sous environnement Learning Labs).

Dans une situation plus défavorable, l'enseignant concepteur s'aide des outils plus classiques de conception de pages Web (Dreamweaver ou autres Word - module Aménagement de LET'S), qui, du fait de leur orientation originale, ne répondent pas directement à toutes les attentes du didacticien et limitent d'autant la profondeur pédagogique du produit final.

Dans un même ordre d'idées, la phase de réalisation informatique peut elle-même être dévolue totalement à des informaticiens, suivant un cahier des charges défini par l'équipe d'enseignants concepteurs. Les produits finis sont alors d'une qualité graphique généralement supérieure, comme le démontrent à la fois le campus Pegasus et le module "mobilité et logistique intégrale» développé à Santander. Notons que ces modules recourent à la même plateforme (WebCT), dont le coût important d'acquisition, accepté par l'institution, en limite par ailleurs la diffusion. 

et l'organisation pédagogique des matériaux. Dans notre étude, il ressort clairement que l'expertise technique - et le temps accordé aux tâches de conception/rédaction conditionne l'organisation pédagogique des matériaux. La mise en ligne de documents de référence est plus fréquemment le fruit d'équipes où ne figurent pas (ou très peu) d'informaticiens. En revanche, l'organisation des matériaux selon des séquences pédagogiques correspond à des équipes renforcées en matière de compétence informatique (Pegasus $\left.{ }^{24}\right)$. Une remarque similaire s'impose si l'on considère l'organisation des tâches d'évaluation et de suivi pédagogique.

Ajoutons enfin que le facteur institutionnel peut influer sur l'ergonomie visuelle des sites. L'unité graphique et structurelle de Pegasus, requise pour identifier clairement l'ensemble des matériaux, ou la politique de normalisation des produits numériques en matière de présentation, menée par les universités de Liège et Santander, par exemple, impliquent des choix que ne maîtrise pas le concepteur. Cette situation explique l'impossibilité pour le programme LET'S de parvenir à une charte graphique commune dans le contexte expérimental qui était le sien.

\subsubsection{Moyens matériels}

Rendre accessibles des matériaux de formation à distance est une chose ; faciliter l'accès à ces matériaux pour les apprenants en est une autre. Si cette remarque peut sembler à la limite du champ que s'est donné cet article, il convient de rappeler cette évidence, car l'expérience montre à quel point l'impact et l'utilité d'une formation à distance sont étroitement liés aux conditions de son accessibilité.

Nous citerons simplement deux exemples à méditer :

- l'une des raisons invoquées par les concepteurs de Pegasus pour l'absence de tout document sonore ou vidéo dans les matériaux (outre l'objectif pédagogique énoncé précédemment) tient à la difficulté technique de télécharger des fichiers sons ou vidéo pour des apprenants ne disposant pas tous, loin s'en faut, de connexions Internet à haut débit. L'arrivée de nouvelles normes, rendant l'échange de tels fichiers plus léger, rendra sous peu caduques ces limites techniques.

- l'expérience pédagogique menée par le présent auteur à l'Université du Havre a montré à quel point il pouvait être difficile, lorsque l'institution ne s'engage pas totalement derrière un tel projet, d'engager les étudiants dans une démarche autonome de travail à distance, en complément du cours en présentiel. Un manque de salles informatiques conduit ces étudiants à utiliser les accès Internet disponibles pour leur propre recherche de stages ou d'information, plus que pour le travail sur la langue qui leur est demandé. Nos contacts avec d'autres centres de langue soulignent que cette situation est loin d'être exceptionnelle. Une question essentielle tient d'ailleurs à la manière de susciter une fréquentation accrue des centres de langues... aux fins qui sont les leurs.

\section{Conclusion}

Quels bénéfices les linguistes peuvent-ils retirer d'une étude pluridisciplinaire?

L'enseignement/apprentissage des langues n'est pas monolithique. Les compétences requises de l'apprenant de langue sont elles-mêmes variées : lexique, grammaire, culture, 
compétences de communication alliant oral et écrit peuvent s'assimiler, à bien des égards, à des approches disciplinaires différentes ${ }^{25}$.

Il semble que les cultures disciplinaires et les facteurs que nous avons mis en évidence dans ces pages jouent un rôle similaire dans la conception de matériaux uniquement destinés aux formations en langue à distance.

Ce que montre notre étude, et dont l'apprentissage des langues peut bénéficier, est la distinction, du fait de l'interaction de ces facteurs multiples, entre des types de matériaux différents, selon la finalité majeure qui leur est attribuée ${ }^{26}$, les moyens techniques, humains et financiers dont disposent les concepteurs.

Plusieurs grandes tendances se dessinent:

- le "polycopié numérique ", pour lequel la finalité référentielle prime et pour lequel l'Internet constitue avant tout une facilité de diffusion.

- les matériaux centrés sur l'activité de l'apprenant, pour lesquels prime la stimulation des processus d'apprentissage. Il s'agit principalement d'activités en ligne (banques d'exercices ou « webquests»).

- certains matériaux accordent une attention particulière aux principes de l'ergonomie pédagogique (fig. 2) en structurant le savoir à acquérir en phases successives reflétant les acquis dans le domaine de l'apprentissage des langues (tutoriels en ligne).

- la combinaison de ces fonctions débouche enfin sur la constitution de matériaux complexes, dans leur structure, qui cherchent à tirer tout le parti possible du support numérique pour «apprendre autrement». Ces matériaux associent références, aides et conseils méthodologiques, contenus disciplinaires, au sein de ce qu'il convient bien d'appeler de véritables environnements d'apprentissages intégrés. C'est tout le sens des expériences menées par le présent auteur à la fois en ce qui concerne le transfert de l'environnement Learning Labs vers le monde de l'Internet et la conception d'un site destiné aux étudiants d'anglais de spécialitée ${ }^{27}$. C'est aussi la raison d'être des plateformes plus ou moins élaborées proposées pour le e-learning.

Ce qui nous semble particulièrement intéressant est la possibilité pour de tels environnements de résoudre l'apparente contradiction entre la volonté d'offrir aux apprenants des matériaux d'une nature nouvelle, tout en accordant au "cours" traditionnel, sur polycopié (fût-il numérisé) une place importante, comme le fait Pegasus. La raison d'être de ces environnements d'apprentissage virtuels est précisément la possibilité qu'ils offrent de générer un réseau original d'interactions entre la discipline cible, l'ordinateur, l'enseignant et l'apprenant. En offrant à ce dernier un savoir atomisé entre des unités indépendantes mais complémentaires, il peut construire, selon des stratégies qui lui sont propres, le savoir qu'il lui faut acquérir. Les interactions hommemachine développent alors conjointement savoirs et savoir-faire.

Ce potentiel pédagogique ne peut toutefois être réalisé que si l'environnement regroupe suffisamment d'outils pour offrir un choix assez large de stratégies d'apprentissage. Expérience et recul théorique sont ainsi d'autant plus importants pour l'équipe de conception que la distance entre le tuteur et l'apprenant rendent difficile, voire impossible, toute communication immédiate.

99 Ce que la présente étude tend à montrer est, dans le cas de la formation ouverte et à distance, le renforcement de la nécessité de réflexion préalable à toute démarche de conception, c'est-à-dire de réflexion accordée au domaine particulier de l'ergonomie pédagogique. 
Enfin, cette étude tend à valider dans ses grandes lignes le modèle global d'ergonomie didactique proposé en fig.1, ainsi que la complémentarité des trois sous-systèmes qui en forment l'ossature (fig. 2 à 4). Il convient toutefois de souligner l'importance, plus encore pour les formations à distance que pour d'autres, de l'élément humain, quelle que soit la forme sous laquelle il peut se décliner :

- introduction dans les matériaux d'une structure de guidage pédagogique implicite ou explicite (Bertin 1998) ;

- mise en place d'un système de tutorat offrant à l'apprenant la possibilité d'aides et conseils que les matériaux ne proposent pas explicitement;

- mise en place d'une structure (réelle ou virtuelle) offrant aux apprenants un espace de contact entre pairs, dont on voit l'importance tant à distance qu'en présentiel.

Le processus de médiation technologique pour la formation en ligne n'occulte donc en rien la place dévolue à l'Homme dans le processus d'enseignement/apprentissage, processus essentiellement fondé sur la communication (de savoirs et de savoir-faire). Reste à savoir jusqu'à quel point cet élément humain peut lui-même être développé par le biais de la simulation dans l'environnement virtuel: une réflexion plus poussée sur la nature et le rôle des agents pédagogiques et sur les modes de guidage à distance apporterait sur ce point des compléments intéressants.

\section{BIBLIOGRAPHIE}

Annoot Emmanuelle et Jean-Claude Bertin. 1997. «L'Intégration des Nouvelles Technologies dans les formations en langue de spécialité (cas des langues du transport et de la logistique) », Rapport du CIRTAI, Plan État - Région, Université du Havre, Mai.

Bertin, Jean-Claude. 1998. " Conception de leçons multimédias : liberté ou guidage ? ", Congrès de la SAES (Société des Anglicistes de l'enseignement supérieur), Mai 1998, publié dans ASp 19-22, 313-31.

Bertin, Jean-Claude. 2001. «L'ergonomie didactique : une approche de la recherche dans le domaine de l'Apprentissage Médiatisé par Ordinateur ». In Mémet M. et M. Petit (dir.) L'anglais de spécialité en France. Bordeaux : Université de Bordeaux II, coll. ASp-GERAS Editeur, 237-254.

Bertin, Jean-Claude. 2001. Des outils pour des langues - Multimédia et apprentissage des langues. Paris : Ellipses.

CIRTAI, Université du Havre (Bertin, Annoot, Gravé). 2003-2004. «Quelle médiation dans les formations à distance avec les nouvelles technologies dans l'Enseignement Supérieur ? Étude des conditions du transfert de ce modèle dans la formation des adultes ", rapport de recherche pour la Maison de la Recherche en Sciences Humaines et Sociales Le Havre - Rouen - Caen. En cours d'élaboration.

Glikman, Viviane. 1999. «Les étudiants face aux médiations technologiques dans les formations ouvertes et à distance ». Actes du $5^{e}$ Colloque Européen sur l'autoformation : «Université ouverte enseignement supérieur et autoformation ", Barcelone, 16-18 Décembre 1999. Paris : L'Harmattan. 
Higgins, John. 1986. « Smart learners and dumb machines ». Systems 14/ 2, 147-50.

Houssaye, Jean 1992 [1988]. Le triangle pédagogique. $2^{\mathrm{e}}$ édition. Berne : Peter Lang SA.

Hubbard, Phillip. 1999. « Teaching agents in CALL tutorials », CALICO'99, Oxford, Ohio, USA.

Hubbard, Phillip. 2000. « Taming teaching agents, meaning technologies and participatory

dramas », CALICO 2000, University of Arizona, Tucson, USA.

Perrin, Michel. 1990. « De l'utilisation communicative des documents authentiques ». In Perrin

M. et L. Delorme (dir.), Langues de spécialité, langues pour le spécialiste : du linguistique au didactique, Actes $d u X^{e}$ Colloque du GERAS, Bordeaux.

Pothier, Maguy. 2003. Multimédias, dispositifs d'apprentissage et acquisition des langues. Paris : Ophrys.

Rabardel, Pierre. 1995. Les Hommes et les Technologies - approche cognitive des instruments contemporains, série Psychologie. Paris : Armand Colin.

Schulze, Mathias. 1999. «Human language technologies and language learning ». European Language Council $2^{\text {nd }}$ Conference, University of Jyväskylä, Finlande.

Tricot, André et Jean-François Rouet. 1998. Les Hypermédias, approches cognitives et ergonomiques. Paris : Hermès.

Uschi, Felix (dir).2003. Language Learning Online: Towards Best Practices. Language Learning Technology Series. Lisse : Swets \& Zeitlinger Publishers.

\section{NOTES}

1. EAO : Enseignement Assisté par Ordinateur. EIAO : Enseignement Intelligemment Assisté par Ordinateur. TELL : Technology Enhanced Language Learning. WELL : Web Enhanced Language Learning 2. Un système-auteur propriétaire permet de créer des applications qui ne pourront être lues par l'ordinateur que si un module de lecture (runtime, système-élève...) y est installé.

3. Un tutoriel est un logiciel éducatif organisé pour guider l'apprenant pas à pas, à l'instar de l'enseignant. Le terme « logiciel éducatif » est plus général.

4. Il s'agit du langage « html » et de ses dérivés.

5. Nous distinguerons le «multimédia» (l'utilisation conjointe, sur support numérique, de médias aussi différents que le texte, l'image, le son ou la vidéo) de l'hypertexte (insertion de liens dynamiques dans un document texte) et de l'hypermédia (insertion de liens dynamiques dans un document constitué de plusieurs médias).

6. Citons par exemple Java, php..

7. Centre Interdisciplinaire de Recherche en Transport et Affaires Internationales : laboratoire de l'Université du Havre appartenant à l'UMR IDEES 6063 du CNRS, auquel est rattaché l'auteur de ces lignes. Associés à ce programme: Emmanuelle Annoot et Patrick Gravé (Sciences de l'Éducation).

8. L'insistance sur la pluralité du facteur humain dans le système en question tient à l'importance et à l'originalité des relations qui s'instaurent entre apprenant et enseignant, d'une part, apprenant et ses pairs, d'autre part, à la différence des systèmes ergonomiques étudiés dans des contextes de production industrielle (Rabardel 1995, par exemple).

9. Une description plus complète de ce modèle est développée dans (Bertin 2001a : 65-83).

10. <http://www.campus-pegasus.org/Accueil/index.htm> L'accès aux matériaux requiert un mot de passe.

11. DAEU : Diplôme d'Accès aux Études Universitaires 
12. < http://www.univ-lehavre.fr/recherche/cirtai/lets/Letshomepage.htm> Il s'agit ici d'une page encore provisoire. L'accès aux matériaux requiert un mot de passe.

13. <http://www.univ-lehavre.fr/boulle/master/pagegenerale.htm>

14. Voir le triangle pédagogique de Houssaye (1988).

15. Pour rendre compte de cette fonction dans un contexte pluridisciplinaire, il suffit de remplacer dans la fig. 1 le terme « langue » par celui de « discipline ».

16. On pourrait ici arguer du fait que l'avocat doit également maîtriser l'oral et qu'il eût donc pu sembler logique de trouver dans ces documents quelques fichiers sonores. Il n'en reste pas moins qu'en droit, le texte est l'unique référence.

17. Courriel : learninglabs@wanadoo.fr - Site Web : <http://www.learninglabs.fr/>

18. Voir sur cet aspect (Bertin 1998).

19. The pedagogue in classical times was the slave who escorted the children to school, an educated slave whose main task was to serve as bodyguard but who could also help the learner in his homework, answer questions, play games, or even give tests. But the pedagogue never initiates; he comes forward when summoned and, when the learner has had enough, he goes back to his place. This is a role which seems in some ways a natural one for the computer to assume. After all, computers were built in the first place to answer questions, not to ask them. (Higgins $1986: 148$ ).

20. Les OCX sont des programmes téléchargeables destinés à être diffusés dans un navigateur Internet. Une fois téléchargés, ces programmes sont installés définitivement sur l'ordinateur.

21. http://ulpmultimedia.u-strasbg.fr/English/Pick\&write_away/

22. Un travail de réflexion sur la composition de ces fichiers de trace est planifié dans le cadre de RANACLES.

23. Notons au passage que la possibilité de reconnaître l'apprenant permet de lui attribuer préférentiellement certains matériaux, en fonction de critères aussi divers que la discipline, l'objectif pédagogique, le niveau... et ceci de manière dynamique. La difficulté des exercices proposés peut, par exemple, varier en fonction de l'évolution des résultats de l'apprenant (système "Cyber Teacher ", utilisé par Télélangues, à la conception duquel l'auteur de ces lignes a collaboré).

24. Notons toutefois l'exception à cette règle que constitue le cas de l'anglais du transport, pour lequel l'auteur, non informaticien de formation, s'investit largement dans un domaine qui constitue son objet d'étude. Le facteur temps est ainsi intégré, sur le plan institutionnel, dans son activité de recherche.

25. La subdivision officieuse de notre $11^{\mathrm{e}}$ section $\mathrm{du}$ CNU ou de la SAES entre linguistique, civilisation, littérature et anglais de spécialité/didactique/nouvelles technologies laisse clairement entendre la distance qui sépare parfois tous les anglisticiens...

26. Une fois mis en ligne, tout matériau devient indépendant de la volonté pédagogique de son créateur. En se l'appropriant, chaque apprenant peut en tirer un profit différent. D'où notre utilisation volontaire de l'adjectif « majeure » dans cette phrase.

27. <http://perso.wanadoo.fr/jean-claude.bertin/SiteBertin.htm>

\section{RÉSUMÉS}

Le recours à l'Internet pour le partage de matériaux pédagogiques et la politique de développement de campus numériques donne à la formation à distance une nouvelle jeunesse. 
Toutefois, le transfert de cours papier vers le support numérique suppose une adaptation, voire une réécriture, qui prend en compte les contraintes et les richesses de ce nouvel environnement. L'enseignement/apprentissage en présentiel médiatisé par l'ordinateur a donné lieu à des tentatives diverses de modélisation. Il s'agit maintenant d'étudier la manière dont la formation ouverte et à distance (FOAD) peut s'inspirer de ces modèles et les faire évoluer. En se fondant sur les exemples de deux campus numériques pluridisciplinaires, l'auteur de cet article cherche à mettre en évidence les principales composantes ergonomiques propres à la formation à distance.

The pooling of resources via the Internet and the development of e-learning has revived the concept of open and distance learning. Yet, the transfer of traditional material to the digital environment supposes a large degree of adaptation to take into account its constraints and originality. Presential CALL has given birth to a number of theoretical models. It is now time to consider how Open and Distance Learning (ODL) can use these models and make them evolve. By considering two cases of pluridisciplinary digital campuses, the author of this paper aims at highlighting the major ergonomic components of distance learning.

\section{INDEX}

Mots-clés : création de matériel pédagogique, ergonomie didactique, FOAD, formation ouverte et à distance, matériel en ligne

Keywords : didactics ergonomics, e-learning material, ODL, open and distance learning, production of educational material

\section{AUTEUR}

\section{JEAN-CLAUDE BERTIN}

Jean-Claude Bertin est professeur à l'Université du Havre, spécialiste des nouvelles technologies pour l'apprentissage des langues. A l'origine du système-auteur Learning Labs, il s'intéresse aux conditions d'intégration des TICE dans les formations. Son approche théorique, baptisée « ergonomie didactique » est développée en particulier dans son ouvrage « Des Outils pour des Langues ", publié chez Ellipses. jean-claude.bertin@unvi-lehavre.fr http://perso.wanadoo.fr/jeanclaude.bertin/SiteBertin.htm 